\title{
PHOTOMETRY OF HICKSON COMPACT GROUP N.90
}

\author{
G. LONGO ${ }^{1}$, H. LORENZ ${ }^{2}$, G. RICHTER ${ }^{2}$ and G. BUSARELLO ${ }^{1}$ \\ 1 Osservatorio Astronomico di Capodimonte, via Moiariello 16, I-80131 Napoli, \\ Italy \\ 2 Astrophysicalisches Institut Potsdam, An der Sternwarte 16, D-14482, \\ Potsdam-Babelsberg, Germany
}

\begin{abstract}
Deep CCD photometry and kinematical observations can be used to constrain the dynamical status of compact groups of galaxies. In the case of Hickson 90 the main interaction is taking place between NGC 7174 and NGC 7173 while NGC 7176 appears to be virtually undisturbed.
\end{abstract}

\section{Introduction}

Recent and independent work by Governato et al. (1991), Zepf \& Whitmore (1991) and Cavaliere et al. (1992) show that in compact groups of galaxies, dynamical friction could be not as effective as previously thought (e.g. White 1991). In particular, if dark matter haloes are properly taken into account, merging of galaxies with comparable masses can take place at the end of a long quasi-stationary phase lasting up to a considerable fraction of the Hubble time. This 'long time scale' scenario can explain some properties of compact groups of galaxies such as, for instance: 1) the large number of groups which is in apparent conflict with the small number of 'ongoing' mergers;

2) the large fraction of groups where the dominant galaxy is of the late morphological types;

3) the finding by Zepf et al. (1991) and Caon et al. (1993) that practically all ellipticals in compact groups have experienced one or more merger events.

One way to further constrain the theory is to obtain detailed photometric and kinematical studies of individual groups. We discuss here the properties of Hickson 90, one of the brightest and nearest groups.

\section{Morphology of Hickson 90}

Hickson 90, also known as Klemola 34, is a compact group formed by four bright NGC objects (NGC 7172, 7173, 7174 and 7176) plus several fainter members. As most other compact groups (Vennik et al. 1993), Hickson 90 appears to be a condensation within a much larger loose group of galaxies (see also Rubin 1974). The brightest and most isolated galaxy in the group is the Seyfert-2, early type spiral NGC 7172, which is crossed by a broad and patchy dust lane slightly warped in the outer regions (Sharples et al. 1984). The other three galaxies in the group, i.e. the 
'subgroup', are embedded in a common, very extended, low surface brightness envelope (Sharples et al. 1984; Longo et al. 1993a). Several tidal features are clearly visible, the most prominent being a faint, broad, 2 ' long plume protruding from NGC 7174 toward NGC 7173, and a more diffuse bi-lobed plume extending for several arcminutes eastward of NGC 7174.

\section{Kinematics of Hickson 90}

Spectra for all galaxies in the group were secured using the ESO $1.5 \mathrm{~m}$. A detailed analysis of the data has been published elsewhere (Longo et al. 1993b). We summarize here only some of the more relevant results:

1) NGC 7176: has quite regular kinematical behaviour and no anomalies can be seen in either the velocity field or in the velocity dispersion profile.

2) NGC 7173: the spectrum taken along the direction of NGC 7174 has the characteristic U-shaped profile produced by the braking action of the tides induced by NGC 7174 on the outer regions of the galaxy (Borne \& Hoessel 1988; Balcells et al. 1989; Madejski et al. 1991).

3) NGC 7174: in spite of having the most distorted morphology among all galaxies in the group, NGC 7174 has a surprisingly normal kinematics with the exception of the data obtained along the direction of NGC 7173, where the interaction is slowing down the outer parts of the galaxy with respect to the inner $(<15$ ") ones.

\section{Photometry of Hickson 90}

Reliable photometry of large nearby galaxies and of groups of galaxies share two problems: targets are usually too large to fit into a single CCD frame, and images need to be corrected for a large number of foreground and background spurious objects. This last problem is particularly relevant for the study of faint outer tidal structures. One additional problem is that group members are usually overlapping and it is quite difficult to derive reliable photometric parameters for individual galaxies.

CCD frames in the BVR and I bands of Hickson 90 were obtained at the $2.2 \mathrm{~m}$ and the NTT ESO-La Silla in the course of three observing runs. Different frames were offcentered and slightly ( $20 \%)$ overlapping. After being smoothed to the same resolution, frames were joined to form a mosaic by matching coordinates and magnitudes for selected stars in the regions of overlap. Final mosaics covered a total field of $18^{\prime} \times 18^{\prime}$.

Bright stars in the fields were eliminated by the procedure described in Lorenz et al. (1993).

The second problem cannot be solved in the general case. In the case of NGC 7174 and NGC 7176 we were fortunate that an independent study of the surface brightness fluctuations (Capaccioli et al. 1993) showed clearly that in the N-W quadrant of NGC 7176 there is little, if any at all, light contribution from NGC 7174. From the N-W quadrant of NGC 7176, we derived non-contaminated luminosity and ellipticity profiles which could be used to model the light distribution of NGC 7176.

The light profile of NGC 7176 looks quite normal for an E0 galaxy and nicely follows an $r^{1 / 4}$ law (Fig. 1a). The model of NGC 7176 was subtracted to obtain the light distribution of NGC 7174 (Fig. 1c). According to predictions from N-body simulations, the isophotes of 

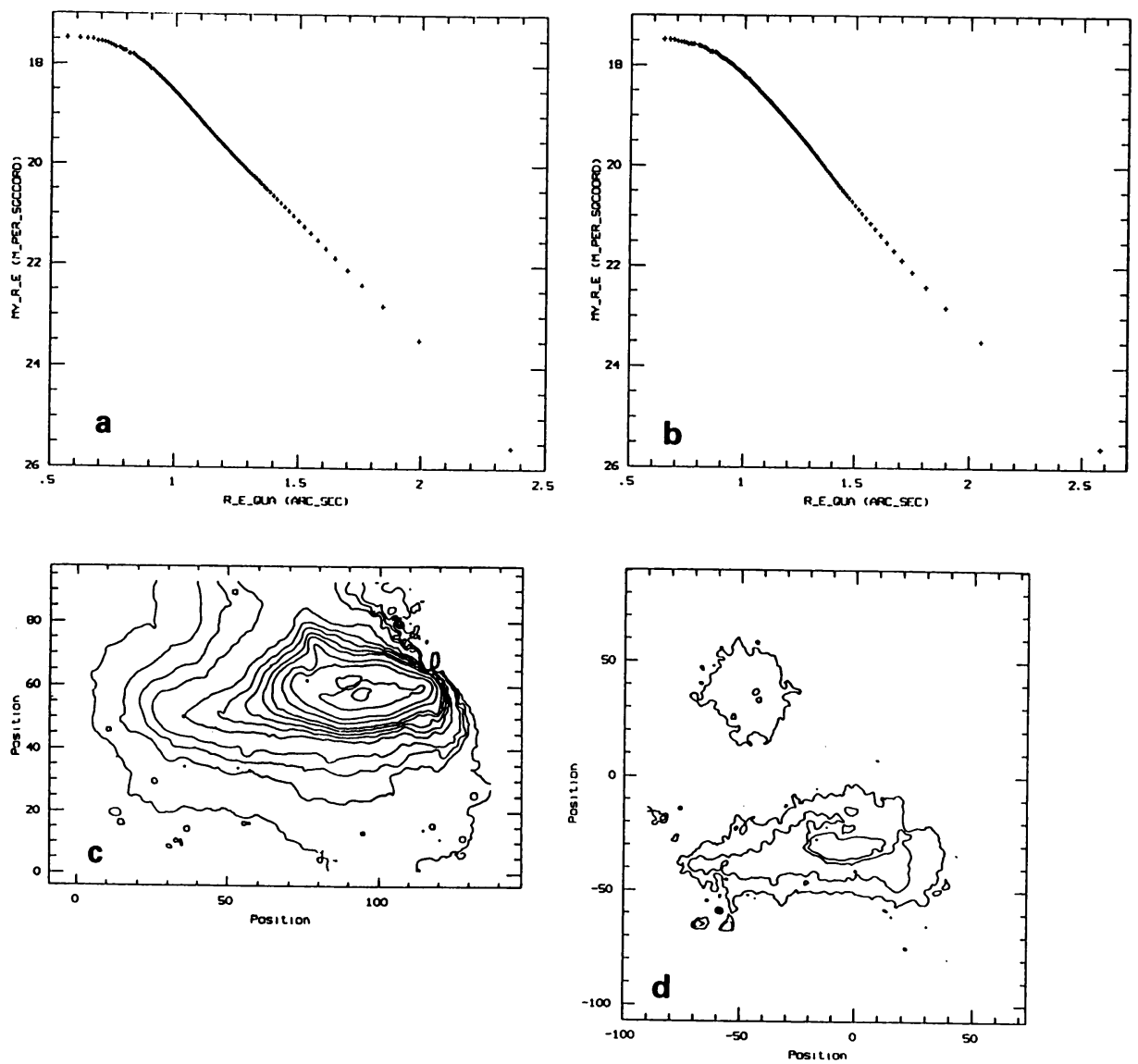

Figure 1. a) Surface brightness profile in the B band for NGC 7176; b) surface brightness profile in the $B$ band for NGC 7173; c) R isophotes for NGC 7174 after the subtraction of the light contribution from NGC 7176; d) (B-R) colour map for the subgroup in Hickson 90.

NGC 7174 turn out to be strongly compressed on the east side.

The light distribution of NGC 7173 was instead derived by using its N-E quadrant. The existence of strong isophotal twisting and of asymmetries in the shape of the light distribution prevented any modeling. It is quite clear, however, that the luminosity profile of NGC 7173 (Fig. 1b) cannot be matched by a single $r^{1 / 4}$ law.

The B, V, R, I frames were used to produce colour maps. The absence of systematic errors was checked against colours obtained by photoelectric multiaperture photometry. No colour gradients are observed in the two early type galaxies NGC 7173 and NGC 7176. The existence of chaotic dust structures makes it quite difficult to understand the structures observed in NGC 7174. 


\section{Some Conclusions}

Hickson 90 is far from equilibrium and the strongest interaction is taking place between NGC 7173 and NGC 7174, while NGC 7176 is almost undisturbed and is lying at some distance from the other galaxies. NGC 7173 is very likely to be triaxial in shape. Our results lead to a $\langle M / L\rangle \simeq 4.5$, ie. to a deficit in the dark matter content in comparison to galaxies isolated or in pairs (see also Rubin et al. 1991). The velocity of the outer parts of NGC 7173 coincides with that of the nucleus of NGC 7174, therefore implying that the tidal torque exerted by NGC 7174 is pulling the outer envelope of NGC 7173. On the other side, the outer envelope of NGC 7174 is pushed toward the observer by the tidal attraction of the elliptical.

\section{References}

Balcells, M., Borne, K.D. and Hoessel, J.G., 1989. Astrophys. J., 336, 655.

Borne, K.D. and Hoessel, J.G., 1988. Astrophys. J., 330, 51 (=BH88)

Caon, N., Capaccioli, M., D'Onofrio, M. and Longo, G., 1993. OAC preprint No. 25.

Capaccioli, M., Lorenz, H., Richter, G. and Bohm, P. 1993. In preparation.

Cavaliere, A., Colafrancesco, S. and Menci, N., 1992. Astrophys. J., 392, 41.

Governato, F., Bathia, R. and Chincarini, G., 1991. Astrophys. J., 371, L15.

Longo, G., Busarello, G., Lorenz, H., Richter, G. and Zaggia S., 1993b. Astron. Astrophys., in press.

Longo, G., Lorenz, G., Richter, G. and Bohm, P., 1993a. In preparation.

Lorenz, H., Richter, G., Capaccioli, M. and Longo, G., 1993. Astron. Astrophys., submitted.

Madejski, R., Bender, R. and Möllenhof, C., 1991. Astron. Astrophys., 242, 58.

Rubin, V.C., Hunter, D.A. and Ford, W.K. jr., 1991. Astrophys. J. Suppl., 76, 153.

Sharples, R.M., Longmore, A.J., Hawarden, T.G. and Carter, D., 1984. Mon. Not. R. astron. Soc., 208, 15.

Vennik, J., Richter, G. and Longo, G. 1993. Astr. Nach. In press.

White, S.D.M., 1991. In 'Dynamics and Interaction of Galaxies', ed. R. Wielen, Springer:Heidelberg, p.380.

Zepf, S.E. and Whitmore, B.C., 1991. Astrophys. J., 383, 524. 